terminatis Secundi Gradus"--consists, as has been said with great truth by Dirichlet, of two distinct parts. Of these the first (Arts. 153-222) contains a complete exposition of the theory of binary quadratic forms, as far as it was known from the researches of Euler and Lagrange ; although even these known results are completed in many respects and are exhibited from a new and independent point of view. The second part (Arts. 223-305) contains investigations which are entirely Gauss's own : the distribution of the classes of binary forms into genera; the determination of the number of ambiguous classes; the demonstration that only one-half of the genera possible a priori actually exist, and the proof of the fundamental theorem deduced from this result; a disquisition on ternary quadratic forms, introduced as a digression; the theory of the decomposition of numbers into three squares; the solution of indeterminate equations of the second degree in rational numbers; the determination of the mean number of the genera and classes; the distinction between regular and irregular determinants. Such is a brief list of the subjects treated of in these marvellous pages, each of which has been the starting-point of long series of important researches by subsequent mathematicians.

In the Additamenta to this section, Gauss intimates that he had succeeded in determining the relations between the determinant and the number of classes; and in a manuscript note he characteristically adds: " $\mathrm{Ex}$ voto nobis sic successit ut nihil amplius desiderandum supersit, Nov. 30-Dec. 3, I800." It is remarkable that he should never have published the wonderful researches to which he here alludes. These researches first saw the light sixty-three years later in the second volume of the collected edition of his works; but the theorem to which they refer had in the interval been rediscovered and demonstrated by Lejeune Dirichlet. The demonstration of Dirichlet had been to a certain extent simplified by M. Hermite, and the form of demonstration found in Gauss's papers after his death approaches very nearly to that adopted by $M$. Hermite.

The sixth section contains some applications of arithmetical principles to various practical questions. Of these the first two are comparatively elementary, and relate to the resolution of fractions into simpler fractions, and to the conversion of vulgar into decimal fractions; the others consist in systematic methods of abbreviating certain tentative processes, such as the solution of quadratic congruences, the decomposition of numbers into their prime factors, the solution of certain indeterminate equations, \&c. The methods of Gauss still remain the least unsatisfactory that have been proposed for the indirect treatment of these difficult problems, of which any direct solution seems impossible.

The seventh section, "De Æquationibus Circuli Sectiones Definientibus," is that which at once made the reputation of the "Disquisitiones Arithmeticæ." It is not too much to say that till the time of Jacobi the profound researches of the fourth and fifth sections were passed over with almost universal neglect. But the well-known theory of the division of the circle comprised in this section was received with great and deserved enthusiasm as a memorable addition to the theory of equations and to the geometry of the circle. One of Gauss's manuscript notes is interesting, "Circulum in 17 partes divisibilem esse geometrice, deteximus I796, Mart. 30," because it shows that he was not yet nineteen when he made this great discovery. Even more remarkable, however, is a passage in the first article of the section (Art. 335), in which Gauss observes that the principles of his method are applicable to many other functions besides the circular functions, and in particular to the transcendents dependent on the integral $\int \frac{d x}{\sqrt{1-x^{4}}}$. This almost casual remark shows (as Jacobi long since observed) that Gauss, at the date of the publication of the "Disquisitiones Arithmeticæ," had already examined the nature and properties of the elliptic functions (the inverses of the elliptic integrals), and had discovered their fundamental property, that of double periodicity. This observation of Jacobi's is amply confirmed by the papers on elliptic transcendents now published in the third volume of Gauss's collected works.

The "Disquisitiones Arithmeticæ" were to have included an eighth section. This eighth section was at first intended to contain a complete theory of congruences, but subsequently Gauss appears to have proposed to continue the work by a more complete discussion of the theory of the division of the circle. Manuscript drafts on each of these subjects were found among his papers; the first of them is especially interesting, as it treats of the general theory of congruences from a point of view closely allied to that subjequently taken by Evariste Galois and by MM. Serret and Dedekind. This draft appears to belong to the years 1797 and 1798 .

To complete this hasty outline of the arithmetical works of Gauss it only remains to mention (I) the remarkable geometrical interpretation of the arithmetical theory of positive binary and ternary quadratic forms, which will be found in his review (1831) of the work of L. Seeber ("Werke," vol. ii. p. I88), and (2) the two important memoirs on the theory of biquadratic residues ( 1825 and 1831). In the second of these memoirs Gauss introduces into arithmetic complex numbers of the form $a+b i$. He finds that in this complex theory every prime number of the form $4 n+I$ is to be regarded as composite, because, being the sum of two squares, e.g. $p=a^{2}+b^{2}$, it is a product of two conjugate factors, $p=(a+b i)(a-b i)$. Thus the true primes of the complex theory may be defined to be the real primes of the form $4 n+3$, and the imaginary factors of real primes of the form $4 n+\mathrm{I}$. Availing himself of this definition, Gauss discovered a theorem of biquadratic reciprocity between any two prime numbers, no less simple than the quadratic law, viz. "If $p_{1}$ and $p_{2}$ are two primary prime numbers, the biquadratic character of $p_{1}$ with regard to $p_{2}$ is the same as that of $p_{2}$ with regard to $p_{1}$."

Both this theorem of reciprocity itself and the introduction of imaginary integers upon which it depends are memorable in the history of arithmetic for the number and variety of the researches to which they have given rise.

It may perhaps seem remarkable that Gauss should have devoted so few memoirs to subjects of an algebraical character. If we except a comparatively unimportant paper on Descartes' rule of signs which appeared in Crelle's Journal in the year I828, his only algebraical memoirs relate to the theorem that every equation has a root. Of this he gave no less than three distinct demonstrations, one in 1799, one in 1815 , and one in 1816 ; the demonstration of 1799 was given in his first published paper-his dissertation as a candidate for the degree of Doctor of Pbilosophy in the University of Göttingen. This demonstration he repeated over again in 1849 , with certain changes and simplification. There can be no question that these three demonstrations are prior to any other, though for various reasons those subsequently given by Cauchy have been justly preferred for the purpose of insertion in our modern text-books.

\section{ANTHROPOLOGY IN AMERICA}

WE cannot speak very highly of Prof. Otis T. Mason's "Account of Progress in Anthropology in the Year I $88 \mathrm{I}$," which was originally embodied in the Smithsonian Report for that year, and is now issued in a separate form. There is no comprehensive survey of the work done in this wide field during the period indicated, and the bibliography, of which the paper mainly consists, is 
vitiated by too many subdivisions. These subdivisions are dealt with in the introluction, where a bewildering scheme of classification is proposed "in order to ascertain the opinion of anthropologists as to its merits." First the science is grouped under three main heads, indicated by terminations furnished by the three Greek words, yoá $\phi \eta$,

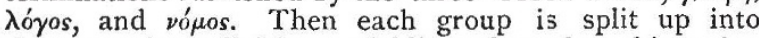
thirteen minor divisions, yielding altogether thirty-nine distinct segmentations, and of course involving the whole subject in dire confusion. The student is expected, for instance, to distingui sh between anthropography, anthropology, and anthroponomy; between pneumatography, pneumatology, and pneumatonomy ; between hexiography, hexiology, hexionomy, and so on. However in the bibliography the author considerately limits himself to eleven headings, which will certainly be amply sufficient to try the patience of those who may have occasion to consult these alphabetical lists. Thus Nesbit's "Antiquity of Man " is entered under Anthropogeny, while Ameghino's "Antiquedad del hombre in La Plata" must be sought for in the section Archaology. These lists should obviously be fused together in one general catalogue, and all the nice subdivisions left to the fancy or ingenuity of the reader. To show their utter absurdity it may suffice to add that under the heading Hexiology there occurs the solitary entry-Buckley, "Climatic Influences on Mankind." Why, it may be asked in conclusion, does B. B. Redding's "Californian Indians and their Food," appear in the section Technology? The interests of science are not furthered by these minute subdivisions and barbarous nomenclatures, which are especially uncalled for in the case of a science whose broad divisions are already marked out with sufficient clearness and accuracy to serve all present practical purposes.

Prof. Mason has been much more usefully employed in the preparation of a series of "Miscellaneous Papers Relating to Anthropology," which also consist of reprints from the Smithsonian Report for 1881. Most of them have reference to the sepulchral mounds, earthworks, fortified lines, shell-heaps, and other remains of prehistoric and historic man so thickly strewn over the Mississippi basin, the eastern States and seaboard of North America. The great number and magnitude of these remains, their universal diffusion over an enormous area, and the character of the objects found in them, all tend to confirm the impression now generally entertained regarding the vast antiquity of man in the New World. On the other hand the views of those anthropologists who still attribute the old works to some superior pre-Columbian race of "mound-builders" distinct from the present aborigines are not strengthened by a more careful examination of these relics. Speaking of the mounds examined by him in Cass County, Illinois, Dr. J. F. Snyder remarks that "the intrinsic evidence of many prehistoric remains of this county sustains their claim to extreme antiquity; but no work or specimen of art of a former race has yet been found here above the capacity or achievement of the typical North American Indian. And in studying the life, habits, and burial customs indicated by these relics, I can see no necessity for ascribing them to the agency of a distinct or superior race, when they express so unmistakably the known status of Indian intellect" (p. 53). This conclusion is amply confirmed by the contents of the enormous shell-heap at Cedar Keys, Florida, which has been carefully examined by Mr. S. T. Walker. Here the pottery found in the successive layers, down to a depth of over twelve feet, shows a continuous advancement in the art from the rude heavy earthenware often mixed with coarse sand or small pebbles occurring in the lowest stratum, through the better finished and slightly ornamented types of the middle stage, to the delicate and beautifully ornamented specimens found near the surface. These objects thus show a progressive improvement upwards, not downwards as would be required by the theory of an extinct
pre-Columbian civilised race, precursor of the present aborigines.

A. H. K.

\section{THE SIZE OF ATOMS 2}

III.

$\mathrm{W}^{\mathrm{E}}$ must then find another explanation of dispersion. I believe there is another explanation. I believe that, while giving up Cauchy's unmodified theory of dispersion, we shall find that the same general principle is applicable, and that by imagining each molecule to be loaded in a certain definite way by elastic connection with heavier matter,-each molecule of the ether to have, in palpable transparent matter, a small fringe, so to speak, of particles, larger and larger in their successive order, elastically connected with it,-we shall have a rude mechanical explanation, realisable by the notably easy addition of the proper appliances to the dynamical models before you, to account for refractive dis. persion in an infinitely fine-grained structure. It is not I7 hours since I saw the possibility of this explanation; I think I now see it perfectly, but you will excuse me not going into the theory more fully under the circumstances. ${ }^{2}$ The difficulty of Cauchy's theory has weighed heavily upon me, when thinking of bringing this subject before you. I could not bring it before you and say there are only four particles in the wave-length, and I could not bring it before you without saying there is some other explanation. I believe another explanation is distinctly to be had in the manner I have slightly indicated.

Now look at those beautiful distributions of colour on the screen before you. They are diffraction spectrums from a piece of glass ruled with 2,000 lines to the inch. And again look; and you see one diffraction spectrum by reflection from one of Rutherford's gratings, in which there are 17,000 lines to the inch on polished speculum-metal. The explanation by "interference", is substantially the same as that which the undulatory theory gives for Newton's rings of light reflected from the two surfaces, which you have already seen. Where light-waves from the apertures between the successive bars of the grating, reach the screen in the same phase, they produce light; there, again, where they are in opposite phases, they produce darkness

The beautiful colours which are produced, depend on the places of conspiring and opposing vibrations on the screen, being different for light waves of different wavelengths; and it is by the measurements of the dimensions of a diffraction spectrum such as the first set you saw (or of finer spectrums from coarser gratings), that Fraunhofer first determined the wave-lengths of the different colours.

I have now, closely bearing on the question of the size of atoms, thanks to Dr. Tyndall, a most beautiful and interesting experiment to show you-the artificial "blue sky," produced by a very wonderful effect of light upon matter, which he discovered. We have here an empty glass tube -it is "optically void." A beam of electric light passes through it now; and you see nothing. Now the light is stopped and we admit vapour of carbon disulphide into the tube. There is now introduced some of this vapour to about 3 inches pressure, and there is also introduced, to the amount of 15 inches pressure, air impregnated with a little nitric acid, making in all rather less than the atmospheric pressure. What is to be illustrated here is the presence of molecules of substances, produced by the decomposition of carbon disulphide by the light.

${ }^{1}$ A lecture delivered by Sir William Thomson at the Royal Institution, on Friday, February 2. Kevised by the Author. Concluded from p. 25.4 2 Farther examination has seemed to me to confirm this first impression and in a paper on the Dynamical Theory of Dispersicn, read before the Roya Society of Edinburgh; on the 5 th of March, I have given a mathematical investigation of the subject.-W. T., March 16, 1883. 